\section{Cancer stem cells predict patient survival}

In animal models of various cancer types, so-called cancer stem cells (CSCs) have been shown to be important for disease maintenance and therapy resistance. However, direct relevance of these findings for human tumors has yet to be demonstrated. In the case of acute myeloid leukemia (AML), Eppert et al. now show that characteristic gene expression profiles of functionally defined CSC populations prognosticate treatment outcome in patients. To obtain CSC expression profiles of human patients, the authors sorted 16 patient samples into four cell populations based on the expression of surface markers, and assayed the ability of each subpopulation to engraft in highly immunodeficient mice. They analyze the gene expression profile of CSC-containing fractions to define a CSC-specific gene signature, which they use to classify AML patients into high-risk and low-risk groups. The high-risk group has an up to 2.4 times higher probability to succumb to the disease. Interestingly, gene signatures derived from cell populations defined on the basis of cell surface markers only (without functional validation) cannot distinguish low-risk from high-risk patients.

(Nat. Med. 17, 1086-1093, 2011)

\section{FcyRllb engagement kills tumors}

The ability of monoclonal antibodies (mAbs) specific for CD40, a member of the tumor necrosis factor receptor (TNFR) family found on antigen-presenting cells (APCs), to induce antitumor immunity has until now been disappointing. Li and Ravetch show that, counterintuitively, mutations in the Fc regions of CD40-binding $\mathrm{mAbs}$ that increase $\mathrm{mAb}$ affinity for the APC Fc $\gamma$ receptor IIb (Fc $\gamma \mathrm{RIIb}$ ) - a receptor previously thought to downregulate activity-stimulate antitumor immunity by activating cytotoxic $\mathrm{T}$ cells. Therapeutic benefits are reflected both by reduced tumor growth and prolonged survival relative to treatment with conventional CD40 mAbs. It remains to be established whether antibodies mutated in their Fc regions to promote $\mathrm{F} c \gamma \mathrm{RIIb}$ engagement couple CD40 and Fc $\gamma$ RIIb molecules on the same or different APCs, whether Fc $\gamma$ RIIb activation can enhance antibody-dependent cellular cytotoxicity triggered by mAbs specific for other TNFR homologs, and what signaling events account for the somewhat unexpected adjuvant effects of engaging inhibitory, but not activating, Fc $\gamma$ receptors. (Science 333, 1030-1034, 2011)

\section{Arming yeast for synthetic biology}

For eukaryotes, synthetic biologists have created individual genes or pathways but not entire genomes. Dymond et al. take a step towards achieving the latter with a paper in Nature that describes the first synthetic eukaryotic chromosome arm, the 90-kb right arm of chromosome
IX in the yeast Saccharomyces cerevisiae. In terms of sheer numbers of base pairs, this DNA molecule is smaller than the 1.08-Mb synthetic prokaryotic genome recently reported, but it was designed with several notable features, including the recoding of all instances of one stop codon to support an expanded genetic code, the addition of unique sequence tags that identify the synthetic DNA, the removal of destabilizing DNA sequences and the strategic scattering of 43 loxP recombination sites throughout the chromosome arm. Synthesis of the DNA itself was reported in Nucleic Acids Research last year. Dymond et al. show that transient expression of the Cre recombinase resulted in extensive deletions and inversions between the loxP sites, generating strain libraries from which auxotrophic mutants could be recovered. (Nature 477, 471-476, 2011; Nucleic. Acids Res. 38, 2594-2602, 2010)

$C M$

\section{Broad-spectrum antiviral strategy}

Most antiviral therapies are pathogen-specific and likely to select for resistance if the virus can mutate the drug target. Rider et al. couple the ability to detect long double-stranded RNA (dsRNA) - a telltale indicator of viral infection in mammalian cells-with procaspaseactivated apoptosis for broad-spectrum antiviral activity. Dubbed DRACOs (double-stranded RNA-activated caspase oligomerizers), these chimeric constructs each comprise (i) a terminal transduction domain for cell penetration, (ii) a dsRNA-binding domain to detect viral infection and (iii) an apoptosis-promoting domain, such as a procaspase-binding domain or a procaspase, which is activated by dsRNA-dependent oligomerization. When tested in 11 mammalian cell types, DRACOs are effective against 15 different viruses. Prophylactic intraperitoneal delivery of DRACOs also reduces the morbidity of mice following subsequent intranasal challenge with H1N1 influenza virus. The strategy seems best suited to situations when exposure to a dangerous virus is anticipated; in contrast, once an infection is established, the treatment may result in potentially dangerous inflammation and organ damage. Unfortunately, the earliest stages of viral infection are usually the most difficult to diagnose. Systemic delivery of DRACOs might also result in nondiscriminate killing of healthy cells infected with nonpathogenic viruses. (PLoS One 6, e22572, 2011)

$\mathrm{PH}$

\section{Motor neurons to order}

In direct lineage conversion, a differentiated cell is converted to another type of differentiated cell through forced expression of a small number of transcription factors. This approach has been used to turn mouse and human fibroblasts into several generic cell types, such as neurons and cardiomyocytes, but little progress has been made in generating more-specialized cell types. A recent study by Son et al. describes the conversion of mouse and human fibroblasts to motor neurons-a neural subtype of special interest because of its role in spinal muscular atrophy and amyotrophic lateral sclerosis. The authors' strategy was to combine transcription factors previously shown to convert fibroblasts to generic neurons with a second set of transcription factors known to be important in motor neuron development. In mouse embryonic fibroblasts, expression of an 11-factor cocktail generated cells resembling motor neurons, and the 11 factors could be whittled down to 7 . These seven factors and one additional factor were then used to generate motor neurons from human fibroblasts. The induced motor neurons resembled their embryo-derived counterparts with respect to morphology, transcriptional profiles, electrophysiological activity, in vivo engraftment and responses to degenerative stimuli. (Cell Stem Cell 9, 205-218, 2011)

$K A$ 